\title{
Interesting Update: A Methodology for the Emulation of Architecture
}

\section{Josiah Carberry}

Department of Psychoceramics, Brown University

http://dx.doi.org/10.5555/26262626x

\section{Interesting Update}

Everything that I claimed in "A Methodology for the Emulation of Architecture" has been reproduced several times by researchers all over the world.

So there.

--Josiah Carberry 